\title{
Displacement exponent for loop-erased random walk on the Sierpiński gasket
}

\author{
Kumiko Hattori
}

August 30, 2021

\begin{abstract}
We prove that loop-erased random walks on finite pre-Sierpiński gaskets can be extended to the infinite pre-Sierpiński gasket by virtue of the 'erasing-larger-loops-first' method, and obtain the asymptotic behavior of the walk as the number of steps increases, in particular, the displacement exponent and a law of the iterated logarithm.
\end{abstract}

Key words: loop-erased random walk ; displacement exponent ; law of the iterated logarithm ; Sierpinski gasket ; fractal

MSC2010 Subject Classifications: 60F99, 60G17, 28A80, 37F25, 37F35

Corresponding author: Kumiko Hattori, khattori@tmu.ac.jp

Department of Mathematics and Information Sciences, Tokyo Metropolitan University, Hachioji, Tokyo 192-0397, Japan.

tel: +81426772475

\section{Introduction}

Loop-erased random walk (LERW) is a process obtained by erasing loops from a simple random walk in chronological order (as soon as each loop is made). LERW was originally considered on $\mathbb{Z}^{d}$ and the existence of the scaling limit has been proved for all $d$. The asymptotic behavior of the walk has been studied in terms of the growth exponent (expected to be the reciprocal of the displacement exponent). For the growth exponents for LERW on $\mathbb{Z}^{d}$, see, for example, [11, [12], [13], [10] and [15].

In this paper, we consider LERW on the Sierpiński gasket and prove the following Theorems $1-3$.

Theorem 1 Loop-erased random walks on the finite Sierpinski gaskets can be extended to a looperased random walk on the infinite Sierpiński gasket.

Let $\lambda=(20+\sqrt{205}) / 15$ and $\nu=\log 2 / \log \lambda$.

Department of Mathematics and Information Sciences, Tokyo Metropolitan University, Hachioji, Tokyo 1920397, Japan. 
Theorem 2 For any $s>0$, there exist positive constants $C_{1}(s)$ and $C_{2}(s)$ such that

$$
C_{1}(s) n^{s \nu} \leqq E\left[|X(n)|^{s}\right] \leqq C_{2}(s) n^{s \nu}
$$

where $X(n)$ denotes the location of the LERW starting at the origin after $n$ steps and $|\cdot|$ the Euclidean distance.

$\nu$ is called the displacement exponent.

Theorem 3 There are positive constants $C_{3}$ and $C_{4}$ such that

$$
C_{3} \leqq \varlimsup_{n \rightarrow \infty} \frac{|X(n)|}{\psi(n)} \leqq C_{4}, \text { a.s. }
$$

where $\psi(n)=n^{\nu}(\log \log n)^{1-\nu}$.

Our main tool for the proof is the 'erasing-larger-loops-first' (ELLF) method, which was introduced to study the scaling limit (the limit as the edge length tends to 0). The scaling limit for LERW on the Sierpinski gasket was obtained by two groups independently, using different methods. For the 'standard' LERW on general graphs, the uniform spanning tree proves to be a powerful tool ([14]). By 'standard', we mean the loops are erased chronologically from a simple random walk as first introduced by G. Lawler ([1]). On the other hand, [4] constructed a LERW on the Sierpiński gasket by ELLF, that is, by erasing loops in descending order of size of loops and proved that the resulting LERW has the same distribution as that of the 'standard' LERW. Futhermore, in [5], it is proved that ELLF does work not only for simple random walks, but also for other kinds of random walks on some fractals, in particular, for self-repelling walks on the Sierpiński gasket introduced in [2]. An important reason for this flexibility is that the ELLF method is based on self-similarity of the Sierpiński gasket.

Another advantage of the ELLF method is facilitate the extension of LERW to the infinite Sierpiński gasket by providing us with a natural definition of two series of probability measures on sets of loopless paths. The extension is not trivial, for the simple random walk on the infinite Sierpinski gasket is recurrent. The exact value of the displacement exponent has been known by a scaling argument ([1]). As for the proof of the existence, the authors erroneously wrote in [4 that Theorem 2 has been proved in [14], however, 14] deals with the scaling limit, not LERW on the infinite Sierpiński gasket, and proves the short-time behavior of the limit process $\bar{X}(t)$ :

Theorem 4 (Theorem 7.10 in [14]) For any $p>0$, there exist constants $C_{5}(p), C_{6}(p)>0$ such that for all $t \in[0,1]$,

$$
C_{5}(p) t^{p \nu} \leqq E\left[|\bar{X}(t)|^{p}\right] \leqq C_{6}(p) t^{p \nu},
$$

where $|\bar{X}(t)|$ denotes the Euclidean distance from the starting point at time $t$ and $\nu=\log 2 / \log \lambda$, $\lambda=(20+\sqrt{205}) / 15$.

It is expected that the same exponent also rules the long-time behavior of the walk, but the method of proof is different, for one has to look into how the scaled number of steps converges, not only the limit distribution. Thus, the author corrects her error and proves Theorem 2 in this paper.

The first mathematical result on the displacement exponent for a non-Markov random walk on the Sierpiński gasket was obtained in [7], dealing with the 'standard' self-avoiding walk, which is defined by the uniform measure on self-avoiding paths of a given length. They showed the existence of the exponent in the form of

$$
\lim _{n \rightarrow \infty} \frac{\log E_{n}\left[\left|X^{\prime}(n)\right|^{s}\right]}{\log n}=s \nu_{S A W}, \quad s>0
$$

where $\left|X^{\prime}(n)\right|$ denotes the end-to-end distance of an $n$-step self-avoiding path, and $\nu_{S A W}=$ $\log 2 / \log \left(\frac{7-\sqrt{5}}{2}\right)$. Since the exponent $\nu_{S A W}$ is different from $\nu$ in Theorem 2, the LERW is in a 
different universality class from the self-avoiding walk. Note that self-avoiding walk cannot be extended to infinite length, for the consistency condition is not satisfied because of culs-de-sac, thus the expectation is taken over the uniform measure on the $n$-step self-avoiding paths. Note also that we have a sharper result in (1.1), which comes from the refinement in the analysis.

The structure of the paper is as follows. In Section 2, we define our notation and in Section 3 , we describe the ELLF method of loop-erasing. Section 4 deals with the asymptotics of the exit times from a series of triangles, which is used in Section 6. In Section 5 we extend the walk to the infinite Sierpiński gasket and finally, in Section [ 6 we prove Theorems 2 and 3.

\section{Random walk on the pre-Sierpiński gaskets}

\subsection{The pre-Sierpiński gaskets}

Let us recall the definition of the pre-Sierpiński gasket: denote $O=(0,0), a_{0}=\left(\frac{1}{2}, \frac{\sqrt{3}}{2}\right), b_{0}=$ $(1,0), a_{N}=2^{N} a_{0}$ and $b_{N}=2^{N} b_{0}$ for $N \in \mathbb{N}$. Let $F_{0}^{\prime}$ be the graph that consists of the three vertices and three edges of $\triangle O a_{0} b_{0}$ and define a recursive sequence of graphs $\left\{F_{N}^{\prime}\right\}_{N=0}^{\infty}$ by

$$
F_{N+1}^{\prime}=F_{N}^{\prime} \cup\left(F_{N}^{\prime}+a_{N}\right) \cup\left(F_{N}^{\prime}+b_{N}\right), \quad N \in \mathbb{Z}_{+}=\{0,1,2, \ldots\},
$$

where $A+a=\{x+a: x \in A\}$ and $k A=\{k x: x \in A\} . F_{0}^{\prime}, F_{1}^{\prime}$ and $F_{2}^{\prime}$ are shown in Fig. 1.
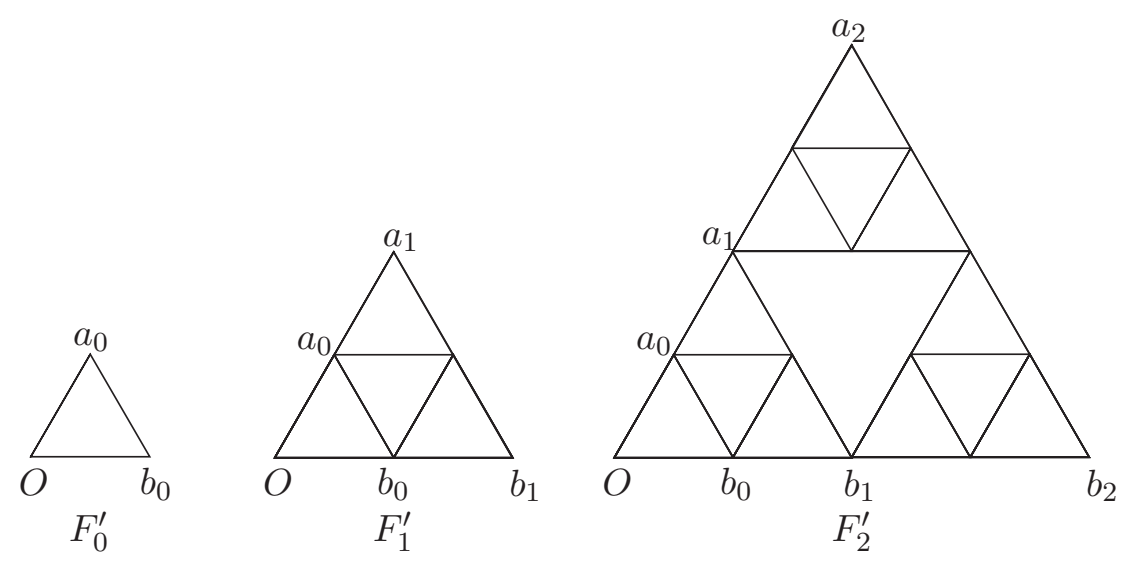

Fig 1: $F_{0}^{\prime}, F_{1}^{\prime}$ and $F_{2}^{\prime}$.

Finally, we let $F_{N}^{\prime R}$ be the reflection od $F_{N}^{\prime}$ with respect to the $y$-axis, and denote $F_{0}=$ $\bigcup_{2=1}^{\infty}$

Furthermore, by letting $G_{0}$ and $E_{0}$ denote the set of vertices and the set of edges of $F_{0}$, respectively, we see that, for each $N \in \mathbb{Z}_{+}, F_{N}=2^{N} F_{0}$ can be regarded as a coarse graph with vertices $G_{N}=\left\{2^{N} x: x \in G_{0}\right\}$ and edges $E_{N}=\left\{2^{N}(x, y):(x, y) \in E_{0}\right\}$. We call an upward (closed and filled) triangle which is a translation of $\triangle O a_{M} b_{M}$ and whose vertices are in $G_{M}$ a $2^{M}$-triangle.

\subsection{Paths on the pre-Sierpiński gaskets}

Let us denote the set of finite paths on $F_{0}$ starting at $O$ by

$$
W=\left\{w=(w(0), w(1), \cdots, w(n)): w(0)=O,(w(i-1), w(i)) \in G_{0}, 1 \leqq i \leqq n, n \in \mathbb{N}\right\} .
$$




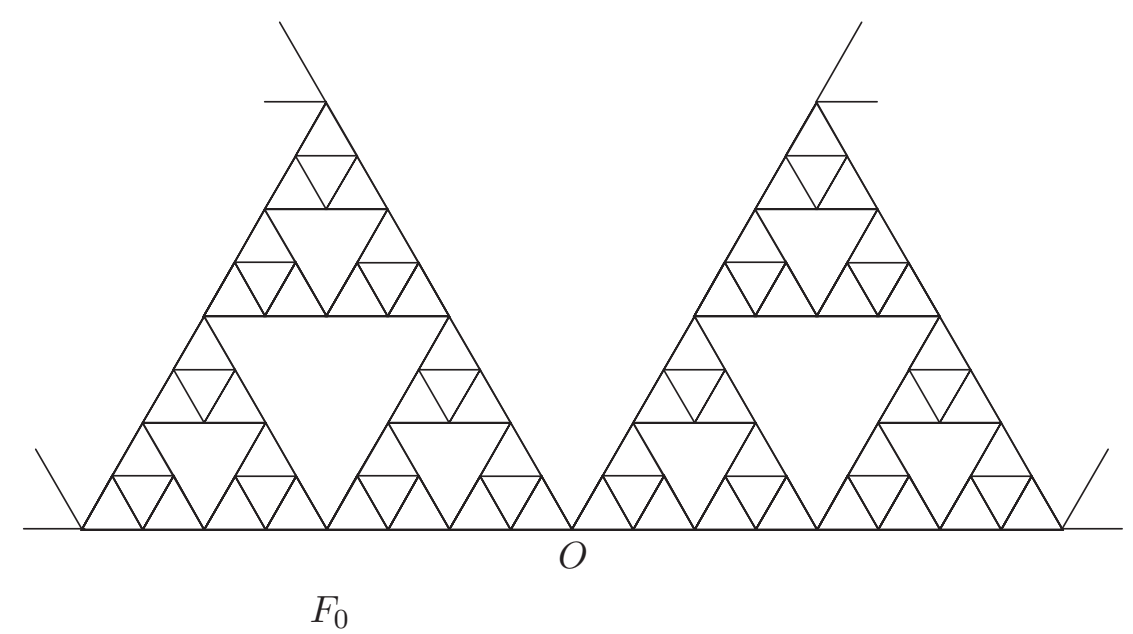

Fig 2: The pre-Sierpiński gasket $F_{0}$.

This gives the natural definition for the length $\ell$ of a path $w=(w(0), w(1), \cdots, w(n)) \in W$; namely, $\ell(w)=n$.

For a path $w \in W$ and $A \subset G_{0}$, we define the hitting time of $A$ by

$$
T_{A}(w)=\inf \{j \geqq 0: w(j) \in A\},
$$

where we set $\inf \emptyset=\infty$. By taking $w \in W$ and $M \in \mathbb{Z}_{+}$, we shall define a recursive sequence $\left\{T_{i}^{M}(w)\right\}_{i=0}^{m}$ of hitting times of $G_{M}$ as follows: Let $T_{0}^{M}(w)=0$, and for $i \geqq 1$, let

$$
T_{i}^{M}(w)=\inf \left\{j>T_{i-1}^{M}(w): w(j) \in G_{M} \backslash\left\{w\left(T_{i-1}^{M}(w)\right)\right\}\right\} ;
$$

here we take $m$ to be the smallest integer such that $T_{m+1}^{M}(w)=\infty$. Then $T_{i}^{M}(w)$ can be interpreted as being the time (steps) taken for the path $w$ to hit vertices in $G_{M}$ for the $(i+1)$-st time, under the condition that if $w$ hits the same vertex in $G_{M}$ more than once in a row, we count it only once.

Now, we consider two sequences of subsets of $W$ as follows: for each $N \in \mathbb{Z}_{+}$, let the set of paths from $O$ to $a_{N}$, which do not hit any other vertices in $G_{N}$ on the way, be

$$
W_{N}=\left\{w=(w(0), w(1), \cdots, w(n)) \in W: w\left(T_{1}^{N}(w)\right)=a_{N}, n=T_{1}^{N}(w)\right\},
$$

and let the set of paths from from $O$ to $a_{N}$ that hit $b_{N}$ 'once' on the way (subject to the counting rule explained above) be

$$
V_{N}=\left\{w=(w(0), w(1), \cdots, w(n)) \in W: w\left(T_{1}^{N}(w)\right)=b_{N}, w\left(T_{2}^{N}(w)\right)=a_{N}, n=T_{2}^{N}(w)\right\} .
$$

Then, for a path $w \in W$ and each $M \in \mathbb{N}$, we define the coarse-graining map $Q_{M}$ by

$$
\left(Q_{M} w\right)(i)=w\left(T_{i}^{M}(w)\right), \quad \text { for } i=0,1,2, \ldots, m
$$

where $m$ is the smallest integer such that $T_{m+1}^{M}(w)=\infty$ as above. Thus,

$$
Q_{M} w=\left(w\left(T_{0}^{M}(w)\right), w\left(T_{1}^{M}(w)\right), \ldots, w\left(T_{m}^{M}(w)\right)\right)
$$

is a path on a coarser graph $F_{M}$. For $w \in W_{N} \cup V_{N}$ and $M \leqq N$, the end point of the coarse-grained path is $w\left(T_{m}^{M}(w)\right)=a_{N}$, and if we write $\left(2^{-M} Q_{M} w\right)(i)=2^{-M} w\left(T_{i}^{M}(w)\right)$, then $2^{-M} Q_{M} w$ is a path in $W_{N-M} \cup V_{N-M}$ and $\ell\left(2^{-M} Q_{M} w\right)=m$. In the following, we often write $w\left(T_{i}^{M}\right)$ instead of $w\left(T_{i}^{M}(w)\right)$. 
Define a family of probability measures $P_{N}$ on $W_{N}, N=1,2, \cdots$ by assigning each $w \in W_{N}$,

$$
P_{N}[w]=\left(\frac{1}{4}\right)^{\ell(w)-1} .
$$

$\left(W_{N}, P_{N}\right)$ defines a family of fixed-end random walks $Z_{N}$ on $F_{N}$ such that

$$
Z_{N}(w)(i)=w(i), \quad i=0, \cdots, \ell(w), \quad w \in W_{N} .
$$

This is a simple random walk on $F_{0}$ starting at $O$ and stopped at the first hitting time of $a_{N}$ conditioned that the walk does not hit any vertices in $G_{N} \backslash\{O\}$ on the way. The factor $(1 / 4)^{-1}$ comes from this conditioning.

Define another family of probability measures $P_{N}^{\prime}$ on $V_{N}, N=1,2, \cdots$. by assigning each $w \in V_{N}$,

$$
P_{N}^{\prime}[w]=\left(\frac{1}{4}\right)^{\ell(w)-2}
$$

$\left(V_{N}, P_{N}^{\prime}\right)$ defines a family of fixed-end random walks $Z_{N}^{\prime}$ on $F_{0}$ such that

$$
Z_{N}^{\prime}(w)(i)=w(i), \quad i=0, \cdots, \ell(w), \quad w \in V_{N} .
$$

This is a simple random walk on $F_{0}$ starting at $O$ and stopped at the first hitting time of $a_{N}$ conditioned that the walk hits $b_{N}$ 'once' on the way.

Note that a coarse grained simple random walk is again a simple random walk on a coarse graph, that is, for $M<N, P_{N} \circ Q_{M}^{-1}=P_{N-M}$ and $P_{N}^{\prime} \circ Q_{M}^{-1}=P_{N-M}^{\prime}$.

\section{Loop erasure by the erasing-larger-loops-first rule}

For $(w(0), w(1), \cdots, w(n)) \in W_{N} \cup V_{N}$, if there are $c \in G_{0}, i$ and $j, 0 \leqq i<j \leqq n$ such that $w(i)=w(j)=c$ and $w(k) \neq c$ for any $i<k<j$, we call the path segment $[w(i), w(i+1), \ldots, w(j)]$ a loop formed at $\mathbf{c}$ and define its diameter by $d=\max _{i \leqq k_{1}<k_{2} \leqq j}\left|w\left(k_{1}\right)-w\left(k_{2}\right)\right|$, where $|\cdot|$ denotes the Euclidean distance. Note that a loop can be a part of another larger loop formed at some other vertex. By definition, the paths in $W_{N} \cup V_{N}$ do not have any loops with diameter greater than $2^{N-1}$. For each $N \in \mathbb{Z}_{+}$, let $\Gamma_{N}$ be the set of loopless paths from $O$ to $a_{N}$ :

$$
\Gamma_{N}=\left\{(w(0), w(1), \cdots, w(n)) \in W_{N} \cup V_{N}: w(i) \neq w(j), 0 \leqq i<j \leqq n, n \in \mathbb{N}\right\} .
$$

Note that any loopless path in $\Gamma_{N}$ is confined in $\triangle O a_{N} b_{N}$.

We shall now describe the loop-erasing procedure in a more organized manner than [4]. We start by erasing loops from paths in $W_{1} \cup V_{1}$.

Loop erasure for $W_{1} \cup V_{1}$

(i) Erase all the loops formed at $O$;

(ii) Progress one step forward along the path, and erase all the loops at the new position;

(iii) Iterate this process, taking another step forward along the path and erasing the loops there, until reaching $a_{1}$.

Denote the resulting path $L w$, where $L: W_{1} \cup V_{1} \rightarrow \Gamma_{1}$ is the loop-erasing operator. Fig. 3 shows all the possible loopless paths from $O$ to $a_{1}$ on $F_{1}$. Here only the parts in $\triangle O a_{1} b_{1}$ are shown, for any path cannot go into the other triangles without making a loop. Note that $w \in W_{1}$ implies $L w \in W_{1} \cap \Gamma_{1}$, but that $w \in V_{1}$ can result in $L w \in W_{1} \cap \Gamma_{1}$, with $b_{1}$ being erased together 


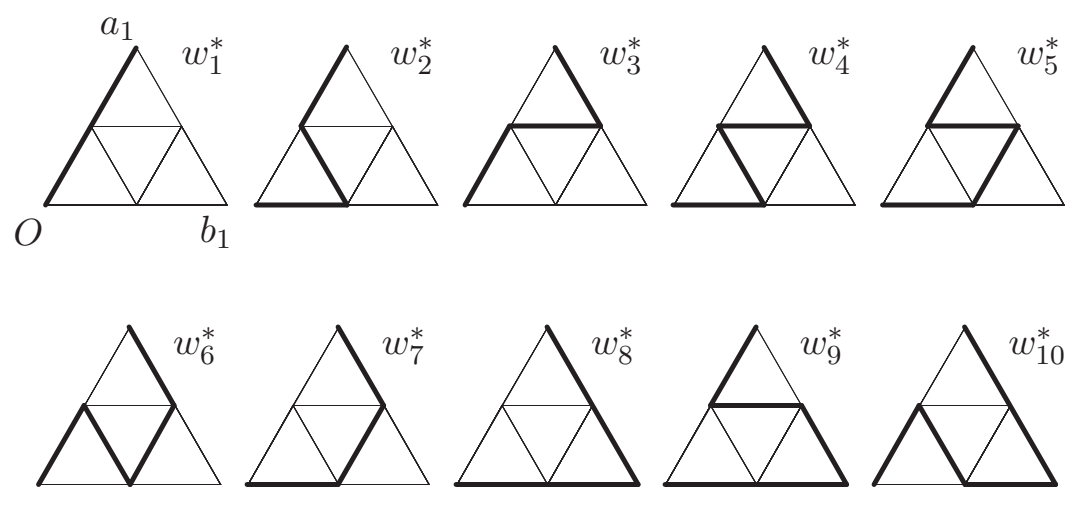

Fig 3: Loopless paths from $O$ to $a_{1}$ on $F_{1}$.

with a loop. So far, our loop-erasing procedure is the same as the chronological method defined for paths on $\mathbb{Z}^{d}$ in $[11$.

For a general $N$, we erase loops from the largest-scale loops down, repeatedly applying the loop-erasing procedure for $W_{1} \cup V_{1}$. To describe the procedure, we introduce a 'step-based' decomposition of a path based on the self-similarity and the symmetries of the pre-Sierpiński gaskets. Assume $w \in W_{N} \cup V_{N}$ and $0 \leqq M<N$. Note that the pair of adjacent $2^{M}$-triangles including $\left(Q_{M} w\right)(i-1),\left(Q_{M} w\right)(i)$ and $\left(Q_{M} w\right)(i+1)$ is similar to $F_{0} \cap\left(\triangle O a_{M} b_{M} \cup \triangle O a_{M}^{R} b_{M}^{R}\right)$, where $\triangle O a_{M}^{R} b_{M}^{R}$ is the reflection of $\triangle O a_{M} b_{M}$ with regard to the $y$-axis. This leads to a unique decomposition:

$$
\left(\tilde{w} ; w_{1}, \cdots, w_{\ell(\tilde{w})}\right), \tilde{w} \in W_{N-M} \cup V_{N-M}, w_{i} \in W_{M}, i=1, \cdots, \ell(\tilde{w})
$$

such that $\tilde{w}$ is similar to $Q_{M} w$ and that the path segment $\left(w\left(T_{i-1}^{M}(w)\right), w\left(T_{i-1}^{M}(w)+1\right)\right), \cdots$, $\left.w\left(T_{i}^{M}(w)\right)\right)$ of $w$ is identified with $w_{i} \in W_{M}$ by appropriate rotation, translation and reflection so that $w\left(T_{i-1}^{M}(w)\right)$ is identified with $O$ and $w\left(T_{i}^{M}(w)\right)$ with $a_{M}$. We shall use this kind of identification throughout the paper. We illustrate a simple example of the decomposition for $N=2$ and $M=1$ in Fig. 4 .

\section{Erasure of the largest loops}

(1) Decompose a path $w \in W_{N} \cup V_{N}$ into $\left(\tilde{w} ; w_{1}, \cdots, w_{\ell(\tilde{w})}\right), \tilde{w}=2^{-(N-1)} Q_{N-1} w \in W_{1} \cup V_{1}$, $w_{i} \in W_{N-1} i=1, \cdots, \ell(\tilde{w})$ as in (3.1) with $M=N-1$. Fig. 5(a) shows the original $w$ and Fig. 5(b) shows $Q_{N-1} w$.

(2) Erase all the loops from $\tilde{w}$ following the loop-erasure for $W_{1} \cup V_{1}$ to obtain $L \tilde{w} \in \Gamma_{1}$. Denote the coarse, loopless path $2^{(N-1)} L \tilde{w}$ on $F_{N-1}$ by $\hat{Q}_{N-1} w$ (Fig. $5(\mathrm{c})$ ).

(3) Restore the original fine structures to the remaining parts as shown in Fig. 5(d) to obtain a path $w^{\prime} \in W_{N} \cup V_{N}$. To be more precise, if we write $\hat{Q}_{N-1} w=\left(w\left(T_{0}^{N-1}\right), w\left(T_{s_{1}}^{N-1}\right), \cdots\right.$, $\left.w\left(T_{s_{n}}^{N-1}\right)\right)$, then for each $i$, between $w\left(T_{s_{i}}^{N-1}\right)$ and $w\left(T_{s_{i+1}}^{N-1}\right)$, insert the path segment $w_{s_{i}+1}=$ $\left(w\left(T_{s_{i}}^{N-1}\right), w\left(T_{s_{i}}^{N-1}+1\right), \cdots, w\left(T_{s_{i}+1}^{N-1}\right)\right)$ chosen from the original decomposition in Step (1). Note that $Q_{N-1} w^{\prime}=\hat{Q}_{N-1} w$ holds.

In this stage all the loops with diameter greater than $2^{N-2}$ have been erased. We repeat Procedure (1)-(3) within each $2^{N-1}$-triangle to erase all the loops with diameter greater than $2^{N-3}$, and then within each $2^{N-2}$-triangle, and so on, until there remain no loops.

To describe the procedure more precisely, we prepare another kind of decomposition, a 'trianglebased' decomposition. For $w \in W_{N}$ and $0 \leqq M \leqq N$, we shall define the sequence $\left(\Delta_{1}, \ldots, \Delta_{k}\right)$ 

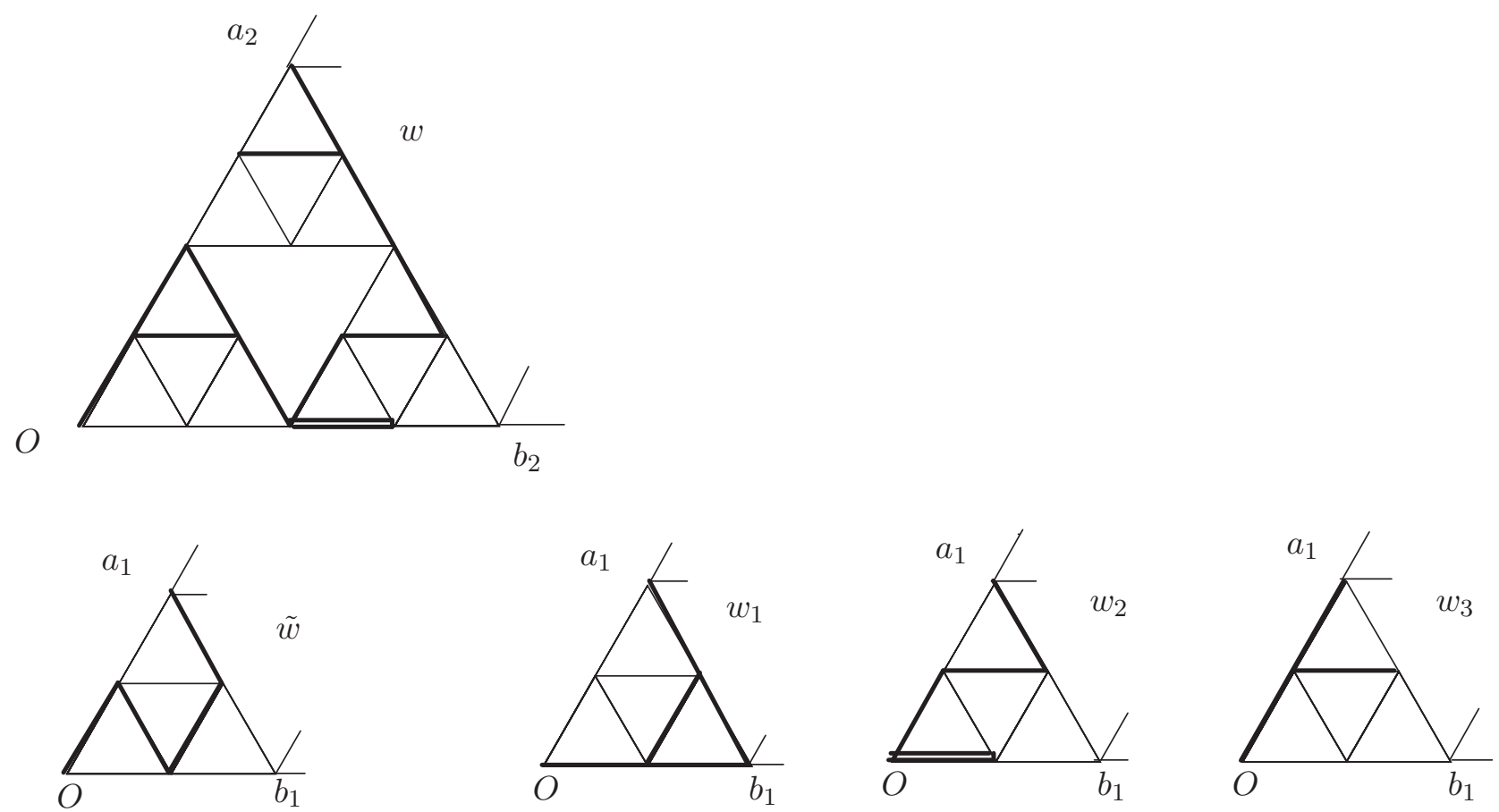

Fig 4: $w, \tilde{w}, w_{1}, w_{2}, w_{3}$.

of the $2^{M}$-triangles $w$ 'passes through', and their exit times $\left\{T_{i}^{e x, M}(w)\right\}_{i=1}^{k}$ as a subsequence of $\left\{T_{i}^{M}(w)\right\}_{i=1}^{m}$ as follows: Let $T_{0}^{e x, M}(w)=0$. There is a unique $2^{M}$-triangle that contains $w\left(T_{0}^{M}\right)$ and $w\left(T_{1}^{M}\right)$, which we denote by $\Delta_{1}$. For $i \geqq 1$, define

$$
J(i)=\min \left\{j \geqq 0: j<m, T_{j}^{M}(w)>T_{i-1}^{e x, M}(w), w\left(T_{j+1}^{M}(w)\right) \notin \Delta_{i}\right\},
$$

if the minimum exists, otherwise $J(i)=m$. Then define $T_{i}^{e x, M}=T_{i}^{e x, M}(w)=T_{J(i)}^{M}(w)$, and let $\Delta_{i+1}$ be the unique $2^{M}$-triangle that contains both $w\left(T_{i}^{e x, M}\right)$ and $w\left(T_{J(i)+1}^{M}\right)$. By definition, we see that $\Delta_{i} \cap \Delta_{i+1}$ is a one-point set $\left\{w\left(T_{i}^{e x, M}\right)\right\}$, for $i=1, \ldots, k-1$. We denote the sequence of these triangles by $\sigma_{M}(w)=\left(\Delta_{1}, \ldots, \Delta_{k}\right)$, and call it the $\mathbf{2}^{\mathbf{M}}$-skeleton of $w$. We call the sequence $\left\{T_{i}^{e x, M}(w)\right\}_{i=0}^{k}$ exit times from the triangles in the skeleton. For each $i$, there is an $n=n(i)$ such that $T_{i-1}^{e x, M}(w)=T_{n}^{M}(w)$. If $T_{i}^{e x, M}(w)=T_{n+1}^{M}(w)$, we say that $\Delta_{i} \in \sigma_{M}(w)$ is Type $\mathbf{1}$, and if $T_{i}^{e x, M}(w)=T_{n+2}^{M}(w)$, Type 2. For $w \in W_{N} \cup V_{N}$ and $M<N$, if $Q_{M} w$ is similar to a path in $\Gamma_{N-M}$, namely, $2^{-M} Q_{M} w \in \Gamma_{N-M}$, then its $2^{M}$-skeleton is a collection of distinct $2^{M}$-triangles and each of them is either Type 1 or Type 2 .

Assume $w \in W_{N} \cup V_{N}$ and $M \leqq N$. For each $\Delta$ in $\sigma_{M}(w)$, the path segment of $\mathbf{w}$ in $\boldsymbol{\Delta}$ is defined by

$$
\left.w\right|_{\Delta}=\left[w(n), T_{i-1}^{e x, M}(w) \leqq n \leqq T_{i}^{e x, M}(w)\right] .
$$

Note that the definition of $T_{i}^{e x, M}(w)$ allows a path segment $\left.w\right|_{\Delta}$ to leak into the neighboring $2^{M}$-triangles. If $Q_{M} w$ is similar to a path in $\Gamma_{N-M}$, then $\left.w\right|_{\Delta} \in W_{M}$ or $\left.w\right|_{\Delta} \in V_{M}$ (identification implied), according to the type of $\Delta \in \sigma_{M}(w)$, where the entrance to $\Delta$ is identified with $O$ and the exit with $a_{M}$. This means that each $w$ such that $Q_{M} w$ is similar to a path in $\Gamma_{N-M}$ can be decomposed uniquely to

$$
\left(\sigma_{M}(w) ;\left.w\right|_{\Delta_{1}}, \cdots,\left.w\right|_{\Delta_{k}}\right),\left.\quad w\right|_{\Delta_{i}} \in W_{M} \cup V_{M}, i=1, \cdots, k .
$$




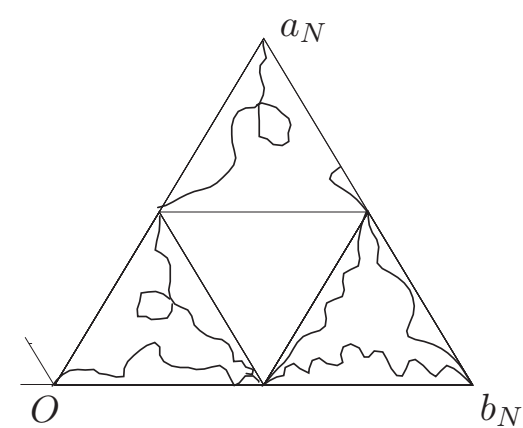

(a)

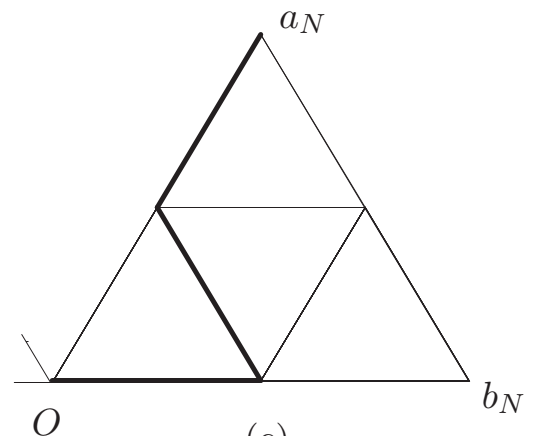

(c)

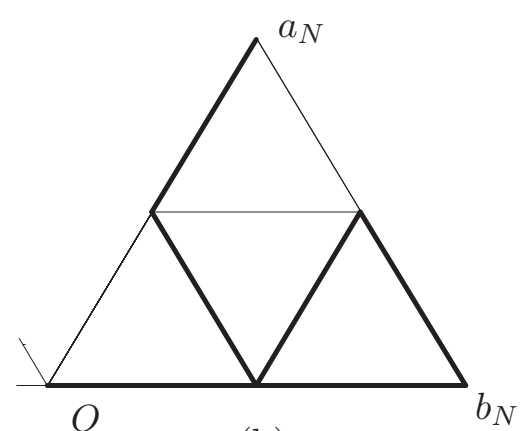

(b)

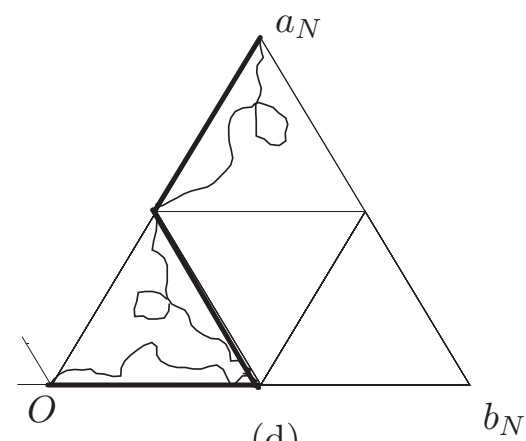

(d)

Fig 5: The loop-erasing procedure: (a) $w$, (b) $Q_{N-1} w$, (c) $\hat{Q}_{N-1} w$, (d) fine structures restored.

We call a loop $\left[w(i), w(i+1), \cdots, w\left(i+i_{0}\right)\right]$ a $\mathbf{2}^{\mathbf{M}}$-scale loop whenever there exists an $M \in \mathbb{Z}_{+}$ such that

$$
\max \left\{N^{\prime}: w(i)=w\left(i+i_{0}\right) \in G_{N^{\prime}}\right\}=M, d \geqq 2^{M},
$$

where $d$ is the diameter of the loop.

\section{Induction step of loop erasure}

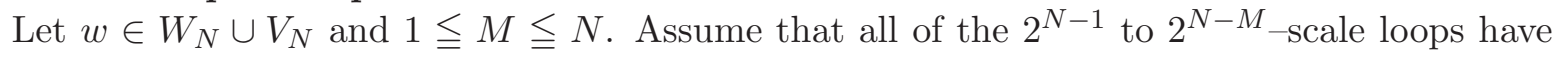
been erased from $w$, and denote the path obtained at this stage by $w^{\prime} \in W_{N} \cup V_{N}$. Note that $Q_{N-M} w^{\prime}$ is similar to a path in $\Gamma_{M}$.

1) Decompose $w^{\prime}$ to obtain $\left(\sigma_{N-M}\left(w^{\prime}\right) ; w_{1}^{\prime}, \cdots w_{k}^{\prime}\right), w_{i}^{\prime} \in W_{N-M} \cup V_{N-M}$ as given in (3.3).

2) From each $w_{i}^{\prime}$, erase $2^{N-M-1}$-scale loops (largest-scale loops) according to the base step procedure (1)-(3) above to obtain $\tilde{w}_{i}^{\prime} \in W_{N-M} \cup V_{N-M}$.

3) Assemble $\left(\sigma_{N-M}\left(w^{\prime}\right) ; \tilde{w}_{1}^{\prime}, \cdots, \tilde{w}_{k}^{\prime}\right)$ to obtain $w^{\prime \prime} \in W_{N} \cup V_{N}$, which is determined uniquely. $w^{\prime \prime}$ has no $2^{N-1}$ to $2^{N-M-1}$-scale loops.

We repeat 1)-3) until we have no loops and denote the resulting loopless path $L w \in \Gamma_{N}$. In this way, the loop erasing operator $L$, first defined for $W_{1} \cup V_{1}$, has been extended to $L$ : $\bigcup_{N=1}^{\infty}\left(W_{N} \cup V_{N}\right) \rightarrow \bigcup_{N=1}^{\infty} \Gamma_{N}$ with $L\left(W_{N} \cup V_{N}\right)=\Gamma_{N}$. Note that the operation described above is essentially a repetition of loop-erasing for $W_{1} \cup V_{1}$.

We induce measures $\hat{P}_{N}=P_{N} \circ L^{-1}$ and $\hat{P}_{N}^{\prime}=P_{N}^{\prime} \circ L^{-1}$, which satisfy $\hat{P}_{N}\left[\Gamma_{N}\right]=\hat{P}_{N}^{\prime}\left[\Gamma_{N}\right]=1$. For $w_{1}^{*}, \cdots, w_{10}^{*}$ shown in Fig. 3, denote

$$
p_{i}=\hat{P}_{1}\left[w_{i}^{*}\right]=P_{1}\left[w: L w=w_{i}^{*}\right], \quad q_{i}=\hat{P}_{1}^{\prime}\left[w_{i}^{*}\right]=P_{1}^{\prime}\left[w: L w=w_{i}^{*}\right] .
$$


They were obtained in [4] by direct calculation:

$$
\begin{aligned}
& \qquad p_{1}=1 / 2, \quad p_{2}=p_{3}=p_{7}=2 / 15, \quad p_{4}=p_{5}=p_{6}=1 / 30, \quad p_{8}=p_{9}=p_{10}=0, \quad \\
& q_{1}=1 / 9, \quad q_{2}=q_{3}=11 / 90, \quad q_{4}=q_{5}=q_{6}=2 / 45, \quad q_{7}=8 / 45, \quad q_{8}=2 / 9, \quad q_{9}=q_{10}=1 / 18 . \\
& \hat{P}_{N} \text { and } \hat{P}_{N}^{\prime} \text { define two kinds of walks } Y_{N}=L Z_{N} \text { and } Y_{N}^{\prime}=L Z_{N}^{\prime} \text { on } F_{0} \cap \triangle O a_{N} b_{N} \text { obtained by } \\
& \text { erasing loops from the simple random walks } Z_{N} \text { and } Z_{N}^{\prime} \text {, respectively. We remark that } \frac{2}{3} \hat{P}_{N}+\frac{1}{3} \hat{P}_{N}^{\prime}
\end{aligned}
$$
equals to the 'standard' LERW studied in [14].

For $w \in W_{N} \cup V_{N}$, we defined $\hat{Q}_{N-1} w$ in Step (2) for the erasure of the largest-scale loops. For later use we define $\hat{Q}_{N-K} w$ on $F_{N-K}$ for all $K=0,1, \cdots, N$. Repeat the induction step 1)-3) $K$ times to have down to $2^{N-K}$-scale loops erased and denote the resulting path $w^{\prime}$. Let $\hat{Q}_{N-K} w=Q_{N-K} w^{\prime}$, namely the coarse path before restoring fine structures. In particular, $\hat{Q}_{N} w=Q_{N} w$ and $\hat{Q}_{0} w=L w$. By construction, the distributions of $2^{-(N-K)} \hat{Q}_{N-K} Z_{N}$ and $2^{-(N-K)} \hat{Q}_{N-K} Z_{N}^{\prime}$ equal to $\hat{P}_{K}$ and $\hat{P}_{K}^{\prime}$, respectively.

\section{Asymptotic behavior of the exit times}

In this section, we look into the asymptotics of exit times $T_{1}^{e x, N}\left(Y_{N}\right)$ and $T_{1}^{e x, N}\left(Y_{N}^{\prime}\right)$ as $N \rightarrow \infty$, which will be used in Section 6 ,

For $w \in \Gamma_{N}$, let us denote the number of $2^{0}$ - triangles of Type 1 (the path passes two of the vertices) and those of Type 2 (the path passes all three vertices) in $\sigma_{0}(w)$ by $s_{1}(w)$ and $s_{2}(w)$, respectively. Note that $T_{1}^{e x, N}(w)=\ell(w)=s_{1}(w)+2 s_{2}(w)$. Define two sequences, $\left\{\Phi_{N}^{(1)}\right\}_{N \in \mathbb{N}}$ and $\left\{\Phi_{N}^{(2)}\right\}_{N \in \mathbb{N}}$, of generating functions by:

$$
\begin{gathered}
\Phi_{N}^{(1)}(x, y)=\sum_{w \in \Gamma_{N}} \hat{P}_{N}(w) x^{s_{1}(w)} y^{s_{2}(w)}, \\
\Phi_{N}^{(2)}(x, y)=\sum_{w \in \Gamma_{N}} \hat{P}_{N}^{\prime}(w) x^{s_{1}(w)} y^{s_{2}(w)}, \quad x, y \geqq 0 .
\end{gathered}
$$

For simplicity, we shall denote $\Phi_{1}^{(1)}(x, y)$ and $\Phi_{1}^{(2)}(x, y)$ by $\Phi^{(1)}(x, y)$ and $\Phi^{(2)}(x, y)$. A crucial observation is that in the process of erasing loops from $Z_{N+1}$, if we stop at the point where we have obtained $\hat{Q}_{1} Z_{N+1}$ after erasing down to $2^{1}$-scale loops, it is nothing but the procedure for obtaining $L Z_{N}$ from $Z_{N}$, namely, the distribution of $2^{-1} \hat{Q}_{1} Z_{N+1}$ equals to $\hat{P}_{N}$. The same holds for $Z_{N+1}^{\prime}$ as well. This combined with (3.4) and (3.5) leads to the recursion relations for the generating functions given below:

Proposition 5 (Proposition 3 in [4])

The above generating functions satisfy the following recursion relations for all $N \in \mathbb{N}$ :

$$
\begin{gathered}
\Phi^{(1)}(x, y)=\frac{1}{30}\left(15 x^{2}+8 x y+y^{2}+2 x^{2} y+4 x^{3}\right) \\
\Phi^{(2)}(x, y)=\frac{1}{45}\left(5 x^{2}+11 x y+2 y^{2}+14 x^{2} y+8 x^{3}+5 x y^{2}\right) \\
\Phi_{N+1}^{(i)}(x, y)=\Phi_{N}^{(i)}\left(\Phi^{(1)}(x, y), \Phi^{(2)}(x, y)\right), \quad i=1,2 .
\end{gathered}
$$


Define the mean matrix by

$$
\mathbf{M}=\left[\begin{array}{cc}
\frac{\partial}{\partial x} \Phi^{(1)}(1,1) & \frac{\partial}{\partial y} \Phi^{(1)}(1,1) \\
\frac{\partial}{\partial x} \Phi^{(2)}(1,1) & \frac{\partial}{\partial y} \Phi^{(2)}(1,1)
\end{array}\right]=\left[\begin{array}{cc}
\frac{9}{5} & \frac{2}{5} \\
\frac{26}{15} & \frac{13}{15}
\end{array}\right]
$$

It is a strictly positive matrix, and the larger eigenvalue is given by $\lambda=(20+\sqrt{205}) / 15=$ $2.2878 \ldots$ The following is a restatement of Proposition 9 in [4].

Proposition 6 (1) Let $G_{N}^{(1)}(t)$ and $G_{N}^{(2)}(t)$ be the Laplace transforms of $\lambda^{-N} T_{1}^{e x, N}\left(Y_{N}\right)$ and $\lambda^{-N} T_{1}^{e x, N}\left(Y_{N}^{\prime}\right)$, respectively, that is,

$$
\begin{gathered}
G_{N}^{(1)}(t)=\hat{E}_{N}\left[\exp \left(-t \lambda^{-N} T_{1}^{e x, N}(w)\right)\right], \\
G_{N}^{(2)}(t)=\hat{E}_{N}^{\prime}\left[\exp \left(-t \lambda^{-N} T_{1}^{e x, N}(w)\right)\right], \quad t \in \mathbb{C}
\end{gathered}
$$

where $\hat{E}_{N}$ and $\hat{E}_{N}^{\prime}$ are expectations with regard to $\hat{P}_{N}$ and $\hat{P}_{N}^{\prime}$, respectively. Then they are expressed in terms of the generating functions as

$$
G_{N}^{(i)}(t)=\Phi_{N}^{(i)}\left(e^{-\lambda^{-N} t}, e^{-2 \lambda^{-N} t}\right) \quad i=1,2 .
$$

(2) $\lambda^{-N} T_{1}^{e x, N}\left(Y_{N}\right)$ and $\lambda^{-N} T_{1}^{e x, N}\left(Y_{N}^{\prime}\right)$ converge in law to some integrable random variables $T_{1}^{*}$ and $T_{2}^{*}$, respectively, as $N \rightarrow \infty$. $T_{1}^{*}$ and $T_{2}^{*}$ have strictly positive probability density functions on $(0, \infty)$.

(3) Let $g_{i}(t)$ be the Laplace transform of $T_{i}^{*}$. For each $i, G_{N}^{(i)}(t)$ converges to $g_{i}(t)$ uniformly on any compact set in $\mathbb{C}$ as $N \rightarrow \infty . g_{1}(t)$ and $g_{2}(t)$ are entire functions on $\mathbb{C}$ and the unique solution to

$$
g_{1}(\lambda t)=\Phi^{(1)}\left(g_{1}(t), g_{2}(t)\right), \quad g_{2}(\lambda t)=\Phi^{(2)}\left(g_{1}(t), g_{2}(t)\right), g_{1}(0)=g_{2}(0)=1 .
$$

To obtain the left tail behavior of the scaled exit times, the following Tauberian theorem has a most suitable form.

Theorem 7 (Theorem 5.9 in [6])

Let $\mu_{N}, N \in \mathbb{N}$ be a family of probability measures on $[0, \infty)$ and let $G_{N}(s)=\int_{0}^{\infty} e^{-s x} \mu_{N}(d x)$, $s>0$ be their Laplace transforms. If there exist positive constants $C_{4.1}-C_{4.4}, s_{0}>0, s_{1} \in \mathbb{R}$ and $0<\nu<1$ such that

$$
C_{4.1} \exp \left(-C_{4.2} s^{\nu}\right) \leqq G_{N}(s) \leqq C_{4.3} \exp \left(-C_{4.4} s^{\nu}\right),
$$

holds for all $s>s_{0}$ and $N>s_{1}+\frac{\nu}{\log 2} \log s$, then the following holds.

(1) There exist positive constants $C_{4.5}$ and $C_{4.6}$ such that for any positive sequence satisfying $\lim _{N \rightarrow \infty} 2^{N(1-\nu) / \nu} \alpha_{N}=\infty$ and $\lim _{N \rightarrow \infty} \alpha_{N}=0$, the following holds:

$$
\begin{aligned}
-C_{4.5} & \leqq \lim _{N \rightarrow \infty} \alpha_{N}^{\nu /(1-\nu)} \log \mu_{N}\left(\left[0, \alpha_{N}\right]\right) \\
& \leqq \varlimsup_{N \rightarrow \infty} \alpha_{N}^{\nu /(1-\nu)} \log \mu_{N}\left(\left[0, \alpha_{N}\right]\right) \leqq-C_{4.6}
\end{aligned}
$$


(2) There exist positive constants $C_{4.7}-C_{4.9}$ such that for any $\xi>0$ and $N \in \mathbb{N}$ satisfying $\left(2^{\frac{1}{\nu}-1}\right)^{N} \xi \geqq C_{4.7}$,

$$
\mu_{N}([0, \xi]) \leqq C_{4.8} e^{-C_{4.9} \xi^{-\nu /(1-\nu)}}
$$

holds.

(1) is a kind of restatement of a Tauberian theorem of exponential type given in [8] and [9], and (2) is the combination of Chebyshev's inequality and (1).

Proposition 8 For $t>0, G_{N}^{(1)}(t)$ and $G_{N}^{(2)}(t)$ satisfy the condition for Theorem $\square$ with $\nu=$ $\log 2 / \log \lambda$.

Proof. Using (4.2), we rewrite the recursion as

$$
G_{N+1}^{(i)}(t)=\Phi^{(i)}\left(G_{N}^{(1)}(t / \lambda), G_{N}^{(2)}(t / \lambda)\right), \quad i=1,2 .
$$

From the explicit form of $\Phi^{(i)}$ in Proposition [5, we have for $0<x, y<1$,

$$
q_{1}(x \wedge y)^{2} \leqq \Phi^{(i)}(x, y) \leqq(x \vee y)^{2}, \quad i=1,2,
$$

where $q_{1}=1 / 9$. Repeating this $M$ times, we have

$$
\left\{q_{1}(x \wedge y)\right\}^{2^{M}} \leqq \Phi_{M}^{(i)}(x, y) \leqq(x \vee y)^{2^{M}}, \quad i=1,2 .
$$

This combined with (4.3) gives

$$
\left\{q_{1}\left(G_{N}^{(1)}\left(t / \lambda^{M}\right) \wedge G_{N}^{(2)}\left(t / \lambda^{M}\right)\right)\right\}^{2^{M}} \leqq G_{N+M}^{(i)}(t) \leqq\left\{G_{N}^{(1)}\left(t / \lambda^{M}\right) \vee G_{N}^{(2)}\left(t / \lambda^{M}\right)\right\}^{2^{M}}
$$

Fix $t_{0}>0$ arbitrarily. Since $\left\{G_{N}^{(1)}\left(t_{0}\right) \vee G_{N}^{(2)}\left(t_{0}\right)\right\}_{N=1}^{\infty}$ and $\left\{\left(G_{N}^{(1)}\left(\lambda t_{0}\right) \wedge G_{N}^{(2)}\left(\lambda t_{0}\right)\right\}_{N=1}^{\infty}\right.$ are positive convergent sequences by Proposition [6 (3), there exist constants $c_{1}, c_{2} \in(0,1)$ such that

$$
q_{1}\left(G_{N}^{(1)}\left(\lambda t_{0}\right) \wedge G_{N}^{(2)}\left(\lambda t_{0}\right)\right)>c_{1}, \quad G_{N}^{(1)}\left(t_{0}\right) \vee G_{N}^{(2)}\left(t_{0}\right)<c_{2},
$$

for all $N \in \mathbb{N}$. For any $t>t_{0}$, choose $M \in \mathbb{Z}_{+}$such that

$$
\lambda^{M} \leqq \frac{t}{t_{0}}<\lambda^{M+1}
$$

Then, the monotonicity of $G_{N}^{(i)}$ combined with (4.5), (4.6) and (4.7) gives

$$
c_{1}^{2^{M}} \leqq G_{N+M}^{(i)}(t) \leqq c_{2}^{2^{M}}, \quad i=1,2
$$

This further leads to

$$
\exp \left(-C_{4.2} t^{\nu}\right) \leqq G_{N}^{(i)}(t) \leqq \exp \left(-C_{4.4} t^{\nu}\right), \quad i=1,2
$$

for all $t>t_{0}$ and $N>\log _{\lambda}\left(t / t_{0}\right)$, where we put $C_{4.2}=-\frac{\log c_{1}}{t_{0}^{\nu}}$ and $C_{4.4}=-\frac{\log c_{2}}{2 t_{0}^{\nu}}$. 


\section{Extention to the infinite Sierpiński gasket}

In this section, we show that the loop-erased random walks defined in Section 3 can be extended to a loop-erased random walk on the infinite Sierpiński gasket. For this purpose, we need walks from $O$ to $b_{N}$ as well as those from $O$ to $a_{N}$. For each $N \in \mathbb{Z}_{+}$, let

$$
\begin{gathered}
W_{N}^{b}=\left\{w=(w(0), w(1), \cdots, w(n)) \in W: w\left(T_{1}^{n}(w)\right)=b_{N}, n=T_{1}^{N}(w)\right\}, \\
V_{N}^{b}=\left\{w=(w(0), w(1), \cdots, w(n)) \in W: w\left(T_{1}^{N}(w)\right)=a_{N}, w\left(T_{2}^{N}(w)\right)=b_{N}, n=T_{2}^{N}(w)\right\} .
\end{gathered}
$$

and probability measures $P_{N}^{(2)}$ on $W_{N}^{b}$ and $P_{N}^{(4)}$ on $V_{N}^{b}$ by

$$
\begin{aligned}
& P_{N}^{(2)}[w]=\left(\frac{1}{4}\right)^{\ell(w)-1}, \quad w \in W_{N}^{b}, \\
& P_{N}^{(4)}[w]=\left(\frac{1}{4}\right)^{\ell(w)-2}, \quad w \in V_{N}^{b} .
\end{aligned}
$$

Let $U_{N}=W_{N} \cup V_{N} \cup W_{N}^{b} \cup V_{N}^{b}$ and extend the loop-erasing operator $L$ to $\bigcup_{N=1}^{\infty} U_{N}$. Denote $P_{N}^{(1)}=P_{N}, P_{N}^{(3)}=P_{N}^{\prime}$ and $\hat{P}_{N}^{(i)}=P_{N}^{(i)} \circ L^{-1}$, for $i=1,2,3,4$. In the rest of the paper, we use the same notation $\Gamma_{N}$ for loopless paths in $U_{N}$. Let

$$
\Omega=\left\{\omega=\left(\omega_{0}, \omega_{1}, \omega_{2}, \cdots\right): \omega_{0} \in \Gamma_{0}, \omega_{N} \in \Gamma_{N},\left.\omega_{N}\right|_{N-1}=\omega_{N-1}, N \in \mathbb{N}\right\},
$$

where $\left.\omega_{N}\right|_{N-1}$ denotes the path $\omega_{N}$ stopped at $T_{1}^{e x, N-1}\left(\omega_{N}\right)$ and $\mathcal{B}$ the $\sigma$-algebra on $\Omega$ generated by cylinder sets. Define the projection onto the first $N+1$ elements by

$$
\pi_{N} \omega=\left(\omega_{0}, \omega_{1}, \ldots, \omega_{N}\right) .
$$

and a probability measure $\tilde{P}_{N}$ on $\pi_{N} \Omega$ by

$$
\tilde{P}_{N}\left[\left(\omega_{1}, \ldots, \omega_{N}\right)\right]=\frac{11}{28}\left(\hat{P}_{N}^{(1)}\left[\omega_{N}\right]+\hat{P}_{N}^{(2)}\left[\omega_{N}\right]\right)+\frac{3}{28}\left(\hat{P}_{N}^{(3)}\left[\omega_{N}\right]+\hat{P}_{N}^{(4)}\left[\omega_{N}\right]\right) .
$$

Proposition 9 The sequence $\left\{\tilde{P}_{N}\right\}, N \in \mathbb{Z}_{+}$defined in (5.1) satisfies:

$$
\tilde{P}_{N}\left[\left(\omega_{0}, \omega_{1}, \ldots, \omega_{N}\right)\right]=\sum_{\omega^{\prime}} \tilde{P}_{N+1}\left[\left(\omega_{0}, \omega_{1}, \ldots, \omega_{N}, \omega^{\prime}\right)\right]
$$

where the sum is taken over all possible $\omega^{\prime} \in \Gamma_{N+1}$ such that $\left.\omega^{\prime}\right|_{N}=\omega_{N}$.

Proof. Assume $u \in U_{N+1}$. Recall that in Step (2) of erasing the largest-scale loops, namely, $2^{N}$-scale loops, from $u$, we obtain $\hat{Q}_{N} u$, which satisfies $2^{-N} \hat{Q}_{N} u \in \Gamma_{1}$ and whose law under $\hat{P}_{N+1}^{(i)}$ is equal to $\hat{P}_{1}^{(i)}$. Let $\Delta_{0}=\triangle O a_{0} b_{0}$ and denote the path segment of $2^{-N} \hat{Q}_{N} u$ in $\Delta_{0}$ by $u_{1}:=\left.\left(2^{-N} \hat{Q}_{N} u\right)\right|_{\Delta_{0}}$. Then $u_{1} \in \Gamma_{0}=\left\{\left(O, a_{0}\right),\left(O, b_{0}\right),\left(O, b_{0}, a_{0}\right),\left(O, a_{0}, b_{0}\right)\right\}$. Denote $v_{1}^{*}=$ $\left(O, a_{0}\right), v_{2}^{*}=\left(O, b_{0}\right), v_{3}^{*}=\left(O, b_{0}, a_{0}\right), v_{4}^{*}=\left(O, a_{0}, b_{0}\right)$, and $\Delta=\triangle O a_{N} b_{N}$. For $\hat{w} \in \Gamma_{N}$, we classify the event $\left\{u \in U_{N+1}:\left.L u\right|_{\Delta}=\hat{w}\right\}$ by $u_{1}$. Note that under the condition that $u_{1}=v_{j}^{*}$, the distribution of $\left.L u\right|_{\Delta}$ is equal to $\hat{P}_{N}^{(j)}$. Thus, for $i=1,3$,

$$
\begin{aligned}
\hat{P}_{N+1}^{(i)}[ & \left.w \in \Gamma_{N+1}:\left.w\right|_{\Delta}=\hat{w}\right]=P_{N+1}^{(i)}\left[u \in U_{N+1}:\left.L u\right|_{\Delta}=\hat{w}\right] \\
= & \sum_{j=1}^{4} P_{N+1}^{(i)}\left[\left.L u\right|_{\Delta}=\hat{w} \mid u_{1}=v_{j}^{*}\right] P_{N+1}^{(i)}\left[u_{1}=v_{j}^{*}\right] \\
= & \sum_{j=1}^{4} \hat{P}_{N}^{(j)}[\hat{w}] \hat{P}_{1}^{(i)}\left[v \in \Gamma_{1}:\left.v\right|_{\Delta_{0}}=v_{j}^{*}\right] \\
= & \hat{P}_{N}^{(1)}[\hat{w}] \hat{P}_{1}^{(i)}\left[\left\{w_{1}^{*}, w_{3}^{*}\right\}\right]+\hat{P}_{N}^{(2)}[\hat{w}] \hat{P}_{1}^{(i)}\left[\left\{w_{5}^{*}, w_{7}^{*}, w_{8}^{*}, w_{9}^{*}\right\}\right] \\
& +\hat{P}_{N}^{(3)}[\hat{w}] \hat{P}_{1}^{(i)}\left[\left\{w_{2}^{*}, w_{4}^{*}\right\}\right]+\hat{P}_{N}^{(4)}[\hat{w}] \hat{P}_{1}^{(i)}\left[\left\{w_{6}^{*}, w_{10}^{*}\right\}\right] .
\end{aligned}
$$


Thus, we have

$$
\begin{aligned}
& \hat{P}_{N+1}^{(1)}\left[w \in \Gamma_{N+1}:\left.w\right|_{\Delta}=\hat{w}\right]=\frac{19}{30} \hat{P}_{N}^{(1)}[\hat{w}]+\frac{1}{6} \hat{P}_{N}^{(2)}[\hat{w}]+\frac{1}{6} \hat{P}_{N}^{(3)}[\hat{w}]+\frac{1}{30} \hat{P}_{N}^{(4)}[\hat{w}], \\
& \hat{P}_{N+1}^{(3)}\left[w \in \Gamma_{N+1}:\left.w\right|_{\Delta}=\hat{w}\right]=\frac{7}{30} \hat{P}_{N}^{(1)}[\hat{w}]+\frac{1}{2} \hat{P}_{N}^{(2)}[\hat{w}]+\frac{1}{6} \hat{P}_{N}^{(3)}[\hat{w}]+\frac{1}{10} \hat{P}_{N}^{(4)}[\hat{w}],
\end{aligned}
$$

For $i=2$, let $\hat{w}^{R}$ and $v_{i}^{* R}$ be the paths obtained by reflection of $\hat{w}$ and $v_{i}^{*}$ with regard to the line $y=x$, respectively. Then we have

$$
\begin{aligned}
\hat{P}_{N+1}^{(2)}[ & \left.w \in \Gamma_{N+1}:\left.w\right|_{\Delta}=\hat{w}\right]=P_{N+1}^{(2)}\left[u \in U_{N+1}:\left.L u\right|_{\Delta}=\hat{w}\right] \\
= & \sum_{j=1}^{4} P_{N+1}^{(2)}\left[\left.L u\right|_{\Delta}=\hat{w} \mid u_{1}=v_{j}^{*}\right] P_{N+1}^{(2)}\left[u_{1}=v_{j}^{*}\right] \\
= & \sum_{j=1}^{4} P_{N+1}^{(1)}\left[\left.L u\right|_{\Delta}=\hat{w}^{R} \mid u_{1}=v_{j}^{* R}\right] P_{N+1}^{(1)}\left[u_{1}=v_{j}^{* R}\right] \\
= & \hat{P}_{N}^{(2)}\left[\hat{w}^{R}\right] \hat{P}_{1}^{(1)}\left[v \in \Gamma_{1}:\left.v\right|_{\Delta_{0}}=v_{2}^{*}\right]+\hat{P}_{N}^{(1)}\left[\hat{w}^{R}\right] \hat{P}_{1}^{(1)}\left[v \in \Gamma_{1}:\left.v\right|_{\Delta_{0}}=v_{1}^{*}\right] \\
& +\hat{P}_{N}^{(4)}\left[\hat{w}^{R}\right] \hat{P}_{1}^{(1)}\left[v \in \Gamma_{1}:\left.v\right|_{\Delta_{0}}=v_{4}^{*}\right]+\hat{P}_{N}^{(3)}\left[\hat{w}^{R}\right] \hat{P}_{1}^{(1)}\left[v \in \Gamma_{1}:\left.v\right|_{\Delta_{0}}=v_{3}^{*}\right] \\
= & \hat{P}_{N}^{(1)}[\hat{w}] \hat{P}_{1}^{(i)}\left[\left\{w_{5}^{*}, w_{7}^{*}, w_{8}^{*}, w_{9}^{*}\right\}\right]+\hat{P}_{N}^{(2)}\left[\hat{w}^{2}\right] \hat{P}_{1}^{(i)}\left[\left\{w_{1}^{*}, w_{3}^{*}\right\}\right] \\
& +\hat{P}_{N}^{(3)}[\hat{w}] \hat{P}_{1}^{(i)}\left[\left\{w_{6}^{*}, w_{10}^{*}\right\}\right]+\hat{P}_{N}^{(4)}[\hat{w}] \hat{P}_{1}^{(i)}\left[\left\{w_{2}^{*}, w_{4}^{*}\right\}\right] .
\end{aligned}
$$

Thus,

$$
\hat{P}_{N+1}^{(2)}\left[w \in \Gamma_{N+1}:\left.w\right|_{\Delta}=\hat{w}\right]=\frac{1}{6} \hat{P}_{N}^{(1)}[\hat{w}]+\frac{19}{30} \hat{P}_{N}^{(2)}[\hat{w}]+\frac{1}{30} \hat{P}_{N}^{(3)}[\hat{w}]+\frac{1}{6} \hat{P}_{N}^{(4)}[\hat{w}],
$$

Similarly, we have

$$
\hat{P}_{N+1}^{(4)}\left[w \in \Gamma_{N+1}:\left.w\right|_{\Delta}=\hat{w}\right]=\frac{1}{2} \hat{P}_{N}^{(1)}[\hat{w}]+\frac{7}{30} \hat{P}_{N}^{(2)}[\hat{w}]+\frac{1}{10} \hat{P}_{N}^{(3)}[\hat{w}]+\frac{1}{6} \hat{P}_{N}^{(4)}[\hat{w}] .
$$

Thus, we see that

$$
\left(\alpha_{1}, \alpha_{2}, \alpha_{3}, \alpha_{4}\right)=\left(\frac{11}{28}, \frac{11}{28}, \frac{3}{28}, \frac{3}{28}\right)
$$

is the unique choice that satisfies

$$
\sum_{i=1}^{4} \alpha_{i} \hat{P}_{N+1}^{(i)}\left[\left.w\right|_{\Delta}=\hat{w}\right]=\sum_{i=1}^{4} \alpha_{i} \hat{P}_{N}^{(i)}[\hat{w}]
$$

for every $\hat{w} \in \Gamma_{N}, N \in \mathbb{N}$.

Proposition 9 provides a consisitency condition for Kolmogorov's extension theorem, and we have the unique probability measure $P$ on $(\Omega, \mathcal{B})$, such that

$$
P \circ \pi_{N}^{-1}=\tilde{P}_{N}
$$

For any $n \in \mathbb{N}$, take an $N$ satisfying $n \leqq 2^{N}$, then the distribution of the first $n$ steps of the path, $\left.\omega_{N}\right|_{n}$ is uniquely determined independently of $N .(\Omega, \mathcal{B}, P)$ defines a loop-erased random walk $X$ on $F_{0}$ such that for each $\omega=\left(\omega_{1}, \omega_{2}, \cdots\right)$ and $i \in \mathbb{Z}_{+}$,

$$
X(\omega)(i)=\omega_{N}(i), \quad i \leqq 2^{N} .
$$


This completes the proof of Theorem 1 .

\section{Remark}

For $N \in \mathbb{N}$ and $w \in U_{N}$, let $u_{M}=\left.\left(2^{-M} \hat{Q}_{M} w\right)\right|_{\Delta_{0}}, M=0,1, \cdots, N$, where $\hat{Q}_{M}$ is defined at the end of Section 3 and $\Delta_{0}=\triangle O a_{0} b_{0}$. Note that $u_{M} \in \Gamma_{0}$. For any $M \leqq N-1$ and any $x_{k} \in \Gamma_{0}, k=M, M+1, \cdots, N$,

$$
P_{N}^{(i)}\left[u_{M}=x_{M} \mid u_{k}=x_{k}, k=M+1, M+2, \cdots, N\right]=P_{N}^{(i)}\left[u_{M}=x_{M} \mid u_{M+1}=x_{M+1}\right] .
$$

Thus, $P_{N}^{(i)}, i=1,2,3,4, N \in \mathbb{N}$ define a family of backward Markov chains on the state space $\Gamma_{0}=\left\{v_{1}^{*}, v_{2}^{*}, v_{3}^{*}, v_{4}^{*}\right\}$ such that

$$
P_{N}^{(i)}\left[u_{N}=v_{i}^{*}\right]=1
$$

and for $M \leqq N-1$,

$$
P_{N}^{(i)}\left[u_{M}=v_{j}^{*} \mid u_{M+1}=v_{k}^{*}\right]=P_{k j},
$$

where $P_{k j}$ denotes the $(k, j)$ - element of the transition probability matrix

$$
\mathbf{P}=\frac{1}{30}\left[\begin{array}{cccc}
19 & 5 & 5 & 1 \\
5 & 19 & 1 & 5 \\
7 & 15 & 5 & 3 \\
15 & 7 & 3 & 5
\end{array}\right]
$$

$\alpha=\frac{1}{28}(11,11,3,3)$ is the unique invariant probability vector, that is, the unique solution to

$$
\alpha=\alpha P \text {. }
$$

Moreover, for any probability vector $a$, it holds that

$$
\lim _{n \rightarrow \infty} a P^{n}=\alpha .
$$

In terms of the loop-erased walk measures, the above fact can be expressed as

$$
\hat{P}_{N+K}^{(i)}\left[\left.w\right|_{K} \in A_{K}\right]=\sum_{j=1}^{4}\left(P^{N}\right)_{i j} \hat{P}_{K}^{(j)}\left[A_{K}\right],
$$

where for $w \in \Gamma_{N+K},\left.w\right|_{K}$ denotes the path $w$ stopped at $T_{1}^{e x, K}(w)$ and $A_{K} \subset \Gamma_{K}$. Thus, for any probability vector $a$, we have as $N \rightarrow \infty$,

$$
\sum_{i=1}^{4} a_{i} \hat{P}_{N+K}^{(i)}\left[\left.w\right|_{K} \in A_{K}\right] \rightarrow \sum_{i=1}^{4} \alpha_{i} \hat{P}_{K}^{(i)}\left[A_{K}\right] .
$$

In particular, $\frac{1}{6}(2,2,1,1)$ represents the 'standard' LERW studied in [14].

\section{Proof of the theorems}

Let $\mathrm{X}$ be the loop-erased random walk defined in Section 5 and let

$$
\tilde{\Phi}_{N}(x, y)=\frac{11}{14} \Phi_{N}^{(1)}(x, y)+\frac{3}{14} \Phi_{N}^{(2)}(x, y)
$$

where $\Phi_{N}^{(i)}(x, y), i=1,2$ are defined in Section 4 . The laplace transform of $\lambda^{-N} T_{1}^{e x, N}(X)$ is given by

$$
\tilde{g}_{N}(t):=\tilde{\Phi}_{N}\left(e^{-t \lambda^{-N}}, e^{-2 t \lambda^{-N}}\right) .
$$


Define for each $n \in \mathbb{N}$,

$$
D_{n}(X)=\min \left\{M \geqq 0:|X(i)| \leqq 2^{M}, 0 \leqq i \leqq n\right\},
$$

and let $K=K(n)$ be the positive integer such that

$$
\lambda^{K} \leqq n<\lambda^{K+1}
$$

holds.

Proposition 10 (short-path estimate) There exist positive constants $C_{6.1}$ and $C_{6.2}$ such that

$$
P\left[D_{n}(X)<K(n)-M\right] \leqq C_{6.1} e^{-C_{6.2} \lambda^{M}}
$$

holds for any $n, M \in \mathbb{N}$ satisfying $K(n)>M$.

Proof. Take $C_{6.2}>0$ arbitrarily. Since Proposition 6 (3) implies that $\left\{\tilde{g}_{N}(t)\right\}$ is a convergent sequence for any $t \in \mathbb{C}$, we can take $C_{6.1}>0$ such that $\tilde{g}_{N}\left(-C_{6.2}\right)<C_{6.1}$ for all $N \in \mathbb{N}$. By Chebyshev's inequality, we have

$$
\tilde{P}_{N}\left[\lambda^{-N} T_{1}^{e x, N}(X) \geqq \lambda^{M}\right] \leqq \tilde{g}_{N}\left(-C_{6.2}\right) e^{-C_{6.2} \lambda^{M}}<C_{6.1} e^{-C_{6.2} \lambda^{M}} .
$$

This leads to

$$
\begin{aligned}
& P\left[D_{n}(X)<K(n)-M\right] \leqq P\left[T_{1}^{e x, K-M}(X)>n\right] \\
& =\tilde{P}_{K-M}\left[T_{1}^{e x, K-M}(w)>n\right] \\
& \leqq \tilde{P}_{K-M}\left[\lambda^{-(K-M)} T_{1}^{e x, K-M}(w)>\lambda^{M}\right] \\
& \leqq C_{6.1} e^{-C_{6.2} \lambda^{M}} \text {. }
\end{aligned}
$$

Proposition 11 (long-path estimate) There exist $C_{6.3}, C_{6.4}>0$ and $N_{0} \in \mathbb{N}$ such that

$$
P\left[D_{n}(X)>K(n)+M\right] \leqq C_{6.3} e^{-C_{6.4} 2^{M}}
$$

for any $n$ satisfying $K(n) \geqq N_{0}$ and any $M \in \mathbb{N}$.

Proof. First note that

$$
\begin{aligned}
P\left[D_{n}(X)>K(n)+M\right] & \leqq P\left[T_{1}^{e x, K+M}(X)<n\right] \\
& =\tilde{P}_{K+M}\left[T_{1}^{e x, K+M}(w)<n\right] \\
& \leqq \tilde{P}_{K+M}\left[T_{1}^{e x, K+M}(w) \leqq \lambda^{K+1}\right] .
\end{aligned}
$$

Fix $0<\delta<1$ arbitrarily, then

$$
\begin{aligned}
\tilde{P}_{K+M}\left[T_{1}^{e x, K+M}(w) \leqq \lambda^{K+1}\right] & =\sum_{w \in \Gamma_{K+M}, \ell(w) \leqq \lambda^{K+1}} \tilde{P}_{K+M}[w] \\
& \leqq \delta^{-1} \sum_{w \in \Gamma_{K+M}, \ell(w) \leqq \lambda^{K+1}} \tilde{P}_{K+M}[w] \delta^{\ell(w) \lambda^{-(K+1)}} \\
& \leqq \delta^{-1} \tilde{\Phi}_{K+M}\left(\delta^{\lambda^{-(K+1)}}, \delta^{2 \lambda^{-(K+1)}}\right) .
\end{aligned}
$$


Let $t^{\prime}=-\lambda^{-1} \log \delta>0$. Since Proposition 6 (3) implies that $\tilde{\Phi}_{N}\left(\delta^{\lambda^{-(N+1)}}, \delta^{2 \lambda^{-(N+1)}}\right)=\tilde{g}_{N}\left(t^{\prime}\right)$ converges as $N \rightarrow \infty$ to a limit strictly smaller than 1 . we can choose $0<r<1$ and $N_{0} \in \mathbb{N}$ such that

$$
\Phi_{N}^{(i)}\left(\delta^{\lambda^{-(N+1)}}, \delta^{2 \lambda^{-(N+1)}}\right)<r, \quad i=1,2
$$

for all $N \geqq N_{0}$. Thus if $K \geqq N_{0}$,

$$
\tilde{\Phi}_{K+M}\left(\delta^{\lambda^{-(K+1)}}, \delta^{2 \lambda^{-(K+1)}}\right)<\tilde{\Phi}_{M}(r, r) \leqq r^{2^{M}}=e^{-C_{6.4} 2^{M}},
$$

where we used (4.4) in the last inequality and set $C_{6.4}=-\log r$. Taking $C_{6.3}=\delta^{-1}$ completes the proof.

To obtain the displacement exponent, we shall use the following inequality that holds for any $\mathbb{N}$-valued random variable $Y$ and $s>0$ :

$$
s C_{6.5}(s) \sum_{k=1}^{\infty} k^{s-1} P[Y \geqq k] \leqq E\left[Y^{s}\right] \leqq s \sum_{k=1}^{\infty} k^{s-1} P[Y \geqq k]+C_{6.6}(s) .
$$

For $0<s<1, C_{6.5}(s)=1, C_{6.6}(s)=1$, for $s>1, C_{6.5}(s)=\frac{1}{2^{s}}, C_{6.6}(s)=0$ and $C_{6.5}(1)=1$, $C_{6.6}(1)=0$.

Let $\nu=\log 2 / \log \lambda$.

Proposition 12 For any $s>0$, there exist a positive constant $C_{1}(s)$ and $n_{1} \in \mathbb{N}$ such that

$$
E\left[|X(n)|^{s}\right] \geqq C_{1}(s) n^{s \nu}
$$

for all $n>n_{1}$.

Proof. Fix $M_{0} \in \mathbb{N}$ such that $C_{6.1} e^{-C_{6.2} \lambda^{M_{0}}}<1 / 2$, where $C_{6.1}$ and $C_{6.2}$ are as in Proposition 10. Take an $n$ large enough so that $K(n)>M_{0}+2$, where $K(n)$ is as in (6.3). Then

$$
P\left[|X(n)| \leqq 2^{K-M_{0}-2}\right] \leqq P\left[D_{n}<K-M_{0}\right]<\frac{1}{2} .
$$

We give a proof in the case for $s>1$. We make use of (6.5) with $P[|X(n)|>n]=0$ in mind.

$$
\begin{aligned}
E\left[|X(n)|^{s}\right] & \geqq \frac{s}{2^{s}} \sum_{m=0}^{\infty} \sum_{k=2^{m}+1}^{2^{m+1}} k^{s-1} P[|X(n)| \geqq k] \\
& \geqq \frac{s}{2^{s}} \sum_{m=0}^{\infty} \sum_{k=2^{m}+1}^{2^{m+1}}\left(2^{m}\right)^{s-1} P\left[|X(n)|>2^{m+1}\right] \\
& \geqq s \sum_{m=0}^{\infty} 2^{s(m-1)} P\left[|X(n)|>2^{m+1}\right] \\
& \geqq s 2^{-\left(M_{0}+4\right) s} 2^{K s} P\left[|X(n)|>2^{K-M_{0}-2}\right] \\
& =s 2^{-\left(M_{0}+4\right) s} 2^{K s}\left(1-P\left[|X(n)| \leqq 2^{K-M_{0}-2}\right]\right) \\
& \geqq s 2^{-\left(M_{0}+5\right) s-1} 2^{K s} \geqq C_{1}(s) n^{s \nu},
\end{aligned}
$$

where we used (6.6) and set $C_{1}(s)=s 2^{-\left(M_{0}+5\right) s-1}$. The case for $0<s \leqq 1$ can be proved similarly. 
Proposition 13 For any $s>0$, there exist a positive constant $C_{2}(s)$ and $n_{2} \in \mathbb{N}$ such that

$$
E\left[|X(n)|^{s}\right] \leqq C_{2}(s) n^{s \nu}
$$

for all $n>n_{2}$.

Proof. First note that

$$
P\left[|X(n)| \geqq 2^{m}\right] \leqq P\left[D_{n}(X)>m-1\right] .
$$

Assume $K=K(n) \geqq N_{0}$ as in Proposition [1], In the case of $s>1$, making use of (6.5), we have

$$
\begin{aligned}
E\left[|X(n)|^{s}\right] & \leqq s \sum_{m=0}^{\infty} 2^{m} \cdot 2^{(s-1)(m+1)} P\left[|X(n)| \geqq 2^{m}\right] \\
& \leqq s 2^{s-1}\left(\sum_{m=0}^{K+1} 2^{s m} P\left[|X(n)| \geqq 2^{m}\right]+\sum_{m=K+2}^{\infty} 2^{s m} P\left[|X(n)| \geqq 2^{m}\right]\right) \\
& \leqq s 2^{s-1}\left(\sum_{m=0}^{K+1} 2^{s m}+\sum_{m=K+2}^{\infty} 2^{s m} P\left[D_{n}(X)>m-1\right]\right) \quad \text { (use of (6.7)) } \\
& \leqq c_{1}(s) 2^{K s}+s 2^{2 s-1} C_{6.3} 2^{K s} \sum_{\ell=1}^{\infty} 2^{\ell s} e^{-C_{6.4} 2^{\ell}} \quad \text { (Proposition 11) } \\
& \leqq C_{2}(s) n^{s \nu},
\end{aligned}
$$

where $c_{1}(s)$ and $C_{2}(s)$ are positive constants depending only on $s$ and we used the convergence of the series above. The case for $0<s \leqq 1$ can be proved similarly.

Proposition 13 combined with Proposition 12 gives Theorem 2 ,

Now we go on to prove the law of the iterated logarithm. First we prove the upper bound:

Proposition 14 There exists $C_{4}>0$ such that

$$
\varlimsup_{n \rightarrow \infty} \frac{|X(n)|}{\psi(n)} \leqq C_{4}, \quad P-\text { a.s. }
$$

where $\psi(n)=n^{\nu}(\log \log n)^{1-\nu}$.

Proof. Let $\mu_{N}$ be the distribution of $\lambda^{-N} T_{1}^{e x, N}(X)$ under $P$. For each $x>1$ there is a unique integer $N$ such that $2^{N} \leqq x<2^{N+1}$. For $k>0$ satisfying $2^{-N} k \geqq C_{4.7}$, Theorem 7 (2) implies that

$$
\begin{aligned}
P\left[\max _{0 \leqq j \leqq k}|X(j)|>x\right] & \leqq P\left[T_{1}^{e x, N}(X) \leqq k\right] \\
& \left.=\mu_{N}\left(\left[0, \lambda^{-N} k\right]\right]\right) \\
& \leqq C_{4.8} e^{-C_{4.9}\left(x k^{-\nu} / 2\right)^{1 /(1-\nu)}} .
\end{aligned}
$$

Let $\gamma>1$ be arbitrary. For $A>0$, let $x=A \psi\left(\gamma^{m}\right)$ and $k$ be the largest integer that does not exceed $\gamma^{m+1}$. The condition $2^{-N} k \geqq C_{4.7}$ is satisfied for $m$ large enough. Thus, the above inequality leads to

$$
\begin{aligned}
\sum_{m=1}^{\infty} P\left[\max _{\gamma^{m}<j \leqq \gamma^{m+1}}|X(j)|>A \psi\left(\gamma^{m}\right)\right] & \leqq \sum_{m=1}^{\infty} P\left[\max _{0 \leqq j \leqq \gamma^{m+1}}|X(j)|>A \psi\left(\gamma^{m}\right)\right] \\
& \leqq c_{3}+C_{4.8} \sum_{m=1}^{\infty} e^{-C_{4.9}\left(x k^{-\nu} / 2\right)^{1 /(1-\nu)}} \\
& \leqq c_{3}+c_{4} C_{4.8} \sum_{m=1}^{\infty} \frac{1}{m^{\alpha}}
\end{aligned}
$$


for some constants $c_{3}, c_{4}>0$ and $\alpha=C_{4.9}\left(\frac{A}{2 \gamma^{\nu}}\right)^{1 /(1-\nu)}$. The sequence $\sum_{m=1}^{\infty} \frac{1}{m^{\alpha}}$ converges if we take $A$ large enough so that $\alpha>1$. The rest is a usual Borel-Cantelli argument and the statement holds with $C_{4}=A$.

Now we show the lower bound:

Proposition 15 There exists $C_{3}>0$ such that

$$
C_{3} \leqq \varlimsup_{n \rightarrow \infty} \frac{|X(n)|}{\psi(n)}, \quad P \text { - a.s. }
$$

holds.

The proof goes along the line of the argument used in [3], but we need to show how the ELLF constraction enables us to make use of a 'Markov structure' to obtain the result. We use the following lemma:

Lemma 16 (A version of the second Borel-Cantelli Lemma used in [2]) Let $B_{1}, B_{2}, \cdots$ be a sequence of events and assume

$$
P\left[B_{m} \mid B_{m+1}^{c}, B_{m+2}^{c}, \cdots, B_{m+k}^{c}\right]=P\left[B_{m} \mid B_{m+1}^{c}\right],
$$

for all $m, k \in \mathbb{N}$. Then

$$
\sum_{m=1}^{\infty} P\left[B_{m} \mid B_{m+1}^{c}\right]=\infty
$$

implies

$$
P\left[\varlimsup_{m \rightarrow \infty} B_{m}\right]=1
$$

Proof of Proposition 15.

Let $\beta=(1-\nu) / \nu, 0<b<1$ and

$$
A_{M}:=\left\{\lambda^{-M} T_{1}^{e x, M}(X) \leqq(b \log M)^{-\beta}\right\} .
$$

We want to show that for an appropriate choice of $b$,

$$
P\left[\varlimsup_{M \rightarrow \infty} A_{M}\right]=1
$$

holds. Let $S_{j}^{M-1}(X)=T_{j}^{e x, M-1}(X)-T_{j-1}^{e x, M-1}(X), j \in\{1,2,3\}$, then

$$
T_{1}^{e x, M}(X)=\sum_{j=1}^{\left|\sigma_{M-1}\right|} S_{j}^{M-1}(X)
$$

where $\left|\sigma_{M-1}\right|$ denotes the number of $2^{M-1}$-triangles in the $2^{M-1}$-skeleton of $X$ stopped at $T_{1}^{e x, M}(X)$, which is either 2 or 3 . Since the right-hand side contains only $S_{j}^{M-1}, j \in\{1,2,3\}$, we have

$$
P\left[A_{M} \mid A_{M-1}^{c} A_{M-2}^{c}, \cdots, A_{M-k}^{c}\right]=P\left[A_{M} \mid A_{M-1}^{c}\right],
$$

and by repeated use of the definition of conditional probability combined with (6.9), we have

$$
P\left[A_{M} \mid A_{M+1}^{c}, A_{M+2}^{c}, \cdots, A_{M+k}^{c}\right]=P\left[A_{M} \mid A_{M+1}^{c}\right] .
$$


In order to show $\sum_{M=1}^{\infty} P\left[A_{M} \mid A_{M+1}^{c}\right]=\infty$, it is sufficient to show $\sum_{M=1}^{\infty} P\left[A_{M} \cap A_{M+1}^{c}\right]=\infty$. Let $x_{M}=(b \log M)^{-\beta}$, then since $S_{1}^{M}(X)=T_{1}^{e x, M}(X)$,

$$
\begin{aligned}
P\left[A_{M}\right. & \left.\cap A_{M+1}^{c}\right]=\tilde{P}_{M+1}\left[T_{1}^{e x, M}(w) \leqq \lambda^{M} x_{M}, T_{1}^{e x, M+1}(w)>\lambda^{M+1} x_{M+1}\right] \\
& =\sum_{y \leqq \lambda^{M} x_{M}} \tilde{P}_{M+1}\left[T_{1}^{e x, M}(w)=y, \sum_{i \geqq 2} S_{i}^{M}(w)+y>\lambda^{M+1} x_{M+1}\right] \\
& \geqq \sum_{y \leqq \lambda^{M} x_{M}} \tilde{P}_{M+1}\left[T_{1}^{e x, M}(w)=y, S_{2}^{M}+y>\lambda^{M+1} x_{M+1}\right] .
\end{aligned}
$$

Let $\tilde{Z}_{N}$ be the simple random walk $\tilde{P}_{N}$ defines on $F_{0} \cap \triangle O a_{N} b_{N}$. Recall the procedure for loop erasure: after erasing largest-scale loops from $\tilde{Z}_{M+1}$ in Step (2), we get $\hat{Q}_{M} \tilde{Z}_{M+1}$ and the law of $2^{-M} \hat{Q}_{M} \tilde{Z}_{M+1}$ is equal to $\tilde{P}_{1}$. We restore the original fine structures to these remaining parts and continue loop erasure. For each $\Delta_{i}$ in $\sigma_{M}\left(\hat{Q}_{M} \tilde{Z}_{M+1}\right)$, if $\Delta_{i}$ is Type 1 with regard to $\hat{Q}_{M} \tilde{Z}_{M+1}$, the rest of the procedure is the same as loop erasure for $Z_{M}$ (modulo rotation and reflection), and if Type 2, the same as that for $Z_{M}^{\prime}\left(Z_{M}\right.$ and $Z_{M}^{\prime}$ are defined in (2.1) and (2.2)). Conditioned on $\hat{Q}_{M} \tilde{Z}_{M+1}$, parts in different $2^{M}$-triangles are independent. Classifying by the types of $\Delta_{1}$ and $\Delta_{2}$ in $\sigma_{M}\left(\hat{Q}_{M} \tilde{Z}_{M+1}\right)$, we have

$$
\begin{aligned}
\tilde{P}_{M+1}[ & \left.T_{1}^{e x, M}(w)=y, S_{2}^{M}(w)+y>\lambda^{M+1} x_{M+1}\right] \\
= & \hat{P}_{M}\left[T_{1}^{e x, M}(w)=y\right] \hat{P}_{M}\left[T_{1}^{e x, M}(w)+y>\lambda^{M+1} x_{M+1}\right] \tilde{P}_{1}\left[\left\{w_{1}^{*}, w_{5}^{*}, w_{7}^{*}\right\}\right] \\
& +\hat{P}_{M}^{\prime}\left[T_{1}^{e x, M}(w)=y\right] \hat{P}_{M}\left[T_{1}^{e x, M}(w)+y>\lambda^{M+1} x_{M+1}\right] \tilde{P}_{1}\left[\left\{w_{2}^{*}, w_{6}^{*}, w_{10}^{*}\right\}\right] \\
& +\hat{P}_{M}\left[T_{1}^{e x, M}(w)=y\right] \hat{P}_{M}^{\prime}\left[T_{1}^{e x, M}(w)+y>\lambda^{M+1} x_{M+1}\right] \tilde{P}_{1}\left[\left\{w_{3}^{*}, w_{8}^{*}, w_{9}^{*}\right\}\right] \\
& +\hat{P}_{M}^{\prime}\left[T_{1}^{e x, M}(w)=y\right] \hat{P}_{M}^{\prime}\left[T_{1}^{e x, M}(w)+y>\lambda^{M+1} x_{M+1}\right] \tilde{P}_{1}\left[\left\{w_{4}^{*}\right\}\right] .
\end{aligned}
$$

Since $x_{M+1} / x_{M} \rightarrow 1$ as $M \rightarrow \infty$, we can take $0<c<1$ such that $c \lambda^{M+1} x_{M+1}<\lambda^{M} x_{M}$ for all large enough $M$. Then we have

$$
\begin{aligned}
& \sum_{y \leqq \lambda^{M} x_{M}} \hat{P}_{M}\left[T_{1}^{e x, M}(w)=y\right] \hat{P}_{M}\left[T_{1}^{e x, M}(w)+y>\lambda^{M+1} x_{M+1}\right] \\
& \geqq \sum_{c \lambda^{M+1} x_{M+1} \leqq y \leqq \lambda^{M} x_{M}} \hat{P}_{M}\left[T_{1}^{e x, M}(w)=y\right] \hat{P}_{M}\left[T_{1}^{e x, M}(w)+y>\lambda^{M+1} x_{M+1}\right] \\
& \quad \geqq \hat{P}_{M}\left[T_{1}^{e x, M}(w) \in\left[c \lambda^{M+1} x_{M+1}, \lambda^{M} x_{M}\right]\right] \hat{P}_{M}\left[T_{1}^{e x, M}(w)>\lambda^{M+1}(1-c) x_{M+1}\right] .
\end{aligned}
$$

By Proposition 6 (2), $\lambda^{-M} T_{1}^{e x, M}$ under $\hat{P}_{M}$ and under $\hat{P}_{M}^{\prime}$ converge in law to $T_{1}^{*}$ and $T_{2}^{*}$, respectively, as $M \rightarrow \infty$, which combined with the fact that $x_{M} \rightarrow 0$ as $M \rightarrow \infty$ leads to

$$
\begin{aligned}
\hat{P}_{M}\left[T_{1}^{e x, M}(w)>\right. & \left.\lambda^{M+1}(1-c) x_{M+1}\right]=\hat{P}_{M}\left[\lambda^{-M} T_{1}^{e x, M}(w)>\lambda(1-c) x_{M+1}\right] \\
& \geqq \hat{P}_{M}\left[\lambda^{-M} T_{1}^{e x, M}(w)>1\right]>\frac{1}{2} P\left[T_{1}^{*}>1\right]
\end{aligned}
$$

for $M$ large enough.

With similar argument for the other terms, we have

$$
\begin{aligned}
P\left[A_{M}\right. & \left.\cap A_{M+1}^{c}\right] \\
> & a \hat{P}_{M}\left[T_{1}^{e x, M}(w) \in\left[c \lambda^{M+1} x_{M+1}, \lambda^{M} x_{M}\right]\right] \tilde{P}_{1}\left[\left\{w_{1}^{*}, w_{3}^{*}, w_{5}^{*}, w_{7}^{*}, w_{8}^{*}, w_{9}^{*}\right\}\right] \\
& +a \hat{P}_{M}^{\prime}\left[T_{1}^{e x, M}(w) \in\left[c \lambda^{M+1} x_{M+1}, \lambda^{M} x_{M}\right]\right] \tilde{P}_{1}\left[\left\{w_{2}^{*}, w_{4}^{*}, w_{6}^{*}, w_{10}^{*}\right\}\right] \\
= & a \tilde{P}_{M}\left[T_{1}^{e x, M}(w) \in\left[c \lambda^{M+1} x_{M+1}, \lambda^{M} x_{M}\right]\right],
\end{aligned}
$$


where $a=\frac{1}{2}\left(P\left[T_{1}^{*}>1\right] \wedge P\left[T_{2}^{*}>1\right]\right)>0$. Moreover,

$$
\begin{aligned}
\tilde{P}_{M}\left[T_{1}^{e x, M}(w) \in\left[c \lambda^{M+1} x_{M+1}, \lambda^{M} x_{M}\right]\right] & \\
& =\tilde{P}_{M}\left[\lambda^{-M} T_{1}^{e x, M}(w) \in\left[0, x_{M}\right]\right]\left(1-\frac{\tilde{P}_{M}\left[\lambda^{-M} T_{1}^{e x, M}(w) \in\left[0, \lambda c x_{M+1}\right]\right.}{\tilde{P}_{M}\left[\lambda^{-M} T_{1}^{e x, M}(w) \in\left[0, x_{M}\right]\right]}\right) .
\end{aligned}
$$

Since $x_{M} \rightarrow 0$ and $2^{M(1-\nu) / \nu} x_{M} \rightarrow \infty$ as $M \rightarrow \infty$, Theorem 7 (1) with $\alpha_{N}=x_{N}$ implies that for large enough $M$

$$
\tilde{P}_{M}\left[\lambda^{-M} T_{1}^{e x, M}(w) \in\left[0, x_{M}\right]\right] \geqq \exp \left(-C_{4.5} x_{M}^{-1 / \beta}\right)=\exp \left(-C_{4.5} b \log M\right)=\frac{1}{M^{C_{4.5} b}} .
$$

We use Theorem 7 (1) again to have

$$
1-\frac{\tilde{P}_{M}\left[\lambda^{-M} T_{1}^{e x, M}(w) \in\left[0, \lambda c x_{M+1}\right]\right.}{\tilde{P}_{M}\left[\lambda^{-M} T_{1}^{e x, M}(w) \in\left[0, x_{M}\right]\right]} \rightarrow 1
$$

as $M \rightarrow \infty$, thus this factor is greater than $1 / 2$ for large enough $M$. Choose $0<b<1$ so that $C_{4.5} b<1$, then for some $M_{0}>0$ and $c_{5}>0$, it holds that

$$
\sum_{M=1}^{\infty} P\left[A_{M} \cap A_{M+1}^{c}\right] \geqq c_{5}+\frac{1}{2} \sum_{M \geqq M_{0}} \frac{1}{M^{C_{4.5} b}}=+\infty .
$$

Lemma 16 implies that for almost all $\omega \in \Omega$, there exists an increasing sequence $\left\{M_{k}(\omega)\right\}$, $k=1,2, \ldots$ such that

$$
\lambda^{-M_{k}} T_{1}^{e x, M_{k}}(X) \leqq\left(b \log M_{k}\right)^{-\beta}
$$

It follows that for $M_{k} \geqq 3$

$$
M_{k} \geqq \frac{\log T_{1}^{e x, M_{k}}(X)+\beta \log b}{\log \lambda}+\frac{\beta \log \log M_{k}}{\log \lambda} \geqq \frac{\log T_{1}^{e x, M_{k}}(X)+\beta \log b}{\log \lambda},
$$

and for any small $\varepsilon>0$, there exists a $k_{0} \in \mathbb{N}$ such that

$$
\log M_{k} \geqq(1-\varepsilon) \log \log T_{1}^{e x, M_{k}}(X)
$$

holds for all $k \geqq k_{0}$. On the other hand, (6.10) implies

$$
\left|X\left(T_{1}^{e x, M_{k}}(X)\right)\right|=2^{M_{k}} \geqq\left(b \log M_{k}\right)^{1-\nu} M_{k}^{\nu} .
$$

This combined with (6.11) leads to

$$
\varlimsup_{n \rightarrow \infty} \frac{|X(n)|}{\psi(n)} \geqq b^{1-\nu}(1-\varepsilon)^{1-\nu} .
$$

Since $\varepsilon$ is arbitrary, we have proved the proposition with $C_{3}=b^{1-\nu}$.

Proposition 14 combined with Proposition 15 gives Theorem 3.

\section{Acknowledgments}

This work is supported by JSPS KAKENHI Grant Number 16K05210. 


\section{References}

[1] Dhar, D., Dhar, A. : Distribution of sizes of erased loops for loop-erased random walks, Phys. Rev. E 55, R2093-2096 (1997).

[2] Hambly, B. M., Hattori, K., Hattori, T. : Self-repelling walk on the Sierpiński gasket, Probab. Theory Relat. Fields 124, 1-25 (2002).

[3] Hambly, B.M., Kumagai, T. : Fluctuation of the transition density for Brownian motion on random recursive Sierpinski gaskets, Stoc. Process Appl. 92, 61-85 (2001).

[4] Hattori, K., Mizuno, M. : Loop-erased random walk on the Sierpinski gasket, Stoch. Process. Appl. 124, 566-585 (2014)

[5] Hattori, K., Ogo, N., Otsuka, T. : A family of self-avoiding random walks interpolating the loop-erased random walk and a self-avoiding walk on the Sierpinski gasket arXiv:1511.04840

[6] Hattori, T. : Random walks and renormalization group, Kyoritsu Publishing (in Japanese).

[7] Hattori, T., Kusuoka, S. : The exponent for mean square displacement of self-avoiding random walk on Sierpinski gasket, Probab. Theory Relat. Fields 93, 273-284 (1992)

[8] Kasahara, Y. : Tauberian theorems of exponential type, Journal of Mathematics of Kyoto Univeristy 18-2 209-219 (1978).

[9] Kasahara, Y., Kosugi, N. : Remarks on Tauberian theorems of exponential type and Frenchel-Legendre transform, Osaka Journal of Mathematics 39, 613-619 (2002).

[10] Kenyon, R. : The asymptotic determinant of the discrete Laplacian, Acta Math. 185, 239-286 (2000).

[11] Lawler, G. F. : A self-avoiding random walk, Duke Math. J. 47, 655-693 (1980).

[12] Lawler, G.F. : Intersections of random walks, Probability and its Applications. Birkhäuser Boston Inc., Boston , MA, 1991

[13] Lawler, G.F. : The logarithmic correction for loop-erased walk in four dimensions, Proceedings of the Conference in Honor of Jean-Pierre Kahane (Orsay, 1993), J. Fourier Anal. Appl. 347-361 (1995).

[14] Shinoda, M., Teufl, E., Wagner, S. : Uniform spanning trees on Sierpiński graphs, Lat. Am. J. Probab. Math. Stat. 11, 737-780 (2014).

[15] Shiraishi D. : Growth exponent for loop-erased random walk in three dimensions, arXiv:1310.1682 